\title{
Desenvolvimento de Ferramenta para a Inserção com Baixa Perturbação de Proteínas de Membrana em Bicamadas Lipídicas para Simulações de Dinâmica Molecular
}

\author{
Luiz F. C. Zonetti \& Alexandre S. Araújo
}

\section{Introdução}

Para a melhor compreensão dos fenômenos físicos e químicos observados em sistemas biológicos os métodos de simulação e modelagem computacional são, sem dúvida, uma ferramenta poderosa. Estes métodos de simulação complementam e estreitam a ligação entre a teoria e o experimento, contribuindo significativamente para o melhor entendimento dos sistemas investigados. O método de Dinâmica Molecular tornou-se popular e poderoso para o estudo de bicamadas lipídicas e proteínas de membrana na última década devido aos avanços nas áreas de software e hardware. Entretanto, algumas simulações de Dinâmica Molecular deste tipo de sistema podem envolver um número elevado de átomos, o que resulta em tempos de simulação muito altos, o que é altamente indesejável na fase de equilibração do sistema.

Neste trabalho apresentamos o desenvolvimento de um script cuja finalidade é inserir proteínas de membrana em bicamadas lipídicas causando a mínima perturbação possível, de modo a minimizar o tempo de simulação necessário para o reestabelecimento do estado de equilíbrio do sistema. O método foi aplicado na inserção do peptídeo de fusão da proteína $\mathrm{E}$ do vírus da dengue em bicamadas puras e mistas. Os resultados obtidos apontam mínima perturbação na bicamada devido ao processo de inserção do peptídeo.

O método desenvolvido foi desenvolvido baseado nas ideias contidas no trabalho de Christian Kandt1, com a diferença de que em nosso caso não são retiradas moléculas de fosfolipídos da bicamada no processo. Isso é particularmente conveniente em simulações onde é calculado o perfil de energia livre por um caminho de reação usando a técnica de múltiplas janelas, já que as diversas janelas criadas pelo nosso método apresentam o mesmo número de fosfolipídios. São apresentados alguns sistemas gerados e resultados da comparação de propriedades físico-químicas das bicamadas equilibradas usadas como entrada do script e as bicamadas geradas com a proteína já inserida no sistema.

\section{Métodos}

Como a finalidade do script é gerar diferentes janelas para simulações de perfil de energia livre por um caminho de reação, a dinâmica de seu funcionamento consiste na geração, a partir de uma configuração inicial, de configurações onde a distância entre dois grupos de átomos assumem diferentes valores consecutivos. No caso do sistema estudado nesse trabalho o caminho de reação é a distância em z entre o centro de massa (CM) do peptídeo e o centro de massa da bicamada. A seguir descrevemos com detalhes cada etapa do método:

1. Movimenta o peptídeo até a posição definida para a janela em questão

2. Verifica se a distância $\mathrm{z}$ entre o $\mathrm{CM}$ do peptídeo e da bicamada é maior que um 
determinado valor definido pelo usuário. Se for, salva a configuração e passa para a próxima janela, se não, segue para as próximas etapas na mesma janela.

3. Calcula a coordenada $\mathrm{x}$ do centro geométrico do peptídeo e define duas bandas na bicamada selecionando moléculas de fosfolipídeos cujos átomos de fósforo possuem coordenada $\mathrm{x}$ maior ou menor que esse valor.

4. Calcula as dimensões do peptídeo na direção $\mathrm{x}$ e desloca as bandas definidas no passo 3 nessa direção de modo a criar uma a abertura com o tamanho do peptídeo.

5. Inicia a sequência de "fechamento" da bicamada onde em cada passo a abertura criada é diminuída de uma distância determinada pelo usuário seguida por uma simulação de minimização de energia para dissipar possíveis superposições de átomos.

6. O fechamento para quando o RMSD entre a posição original e atual dos átomos de fósforo é mínima, o que significa que a bicamada retornou à conformação mais próxima da original possível.

7. Salva a configuração obtida e passa para a próxima janela.

\section{Detalhes Computacionais}

A linguagem utilizada no script foi o tcl e sua execução é feita pelo software Visual Molecular Dynamics (VMD)2. Como as simulações dos sistemas gerados serão realizadas utilizando o programa NAMD (Nanoscale Molecular Dynamics program $)^{3}$, o scritp trabalha com arquivos dos tipos $p d b$ e $p s f$.

Nos resultados apresentados utilizamos bicamadas lipídicas em solução aquosa compostas por lipídeos de POPC (Palmitoil-Oleil-FosfatidilColina) (bicamada pura) e por lipídeos de POPC e POPG (Palmitoil-Oleil-Fosfatidil-Glicerol) na proporção 4:1 (bicamada mista). Inserimos nessas bicamadas uma sequência de aminoácidos da proteína $\mathrm{E}$ do vírus da dengue do resíduo 98 até o resíduo 110 , conhecido como peptídeo de fusão, que contem a seguinte sequência de aminoácidos $\mathrm{D}$ R G W G N G C G L F G K.
O Perfil de Densidade de Massa fornece informações sobre a espessura da bicamada e a posição média de moléculas específicas como a água, proteína, grupos que compõem os lipídios, etc, para isso usamos o plugin Density Profile do VMD ${ }^{4}$.

\section{Resultados e Discussão}

Os arquivos de entrada para o script (bicamadas lipídicas solvatadas) foram gerados no site do CHARMM$\mathrm{GUI}^{5,6}$ com a ferramenta Membrane Builder ${ }^{7,8}$. Geramos dois sistemas para nossas simulações: a bicamada pura contém 288 moléculas de POPC e a mista contendo 240 moléculas de POPC e 60 moléculas de POPG. As bicamadas foram posicionadas de modo que o eixo normal ao seu plano coincidisse com o eixo z.

As bicamadas foram solvatadas com moléculas de água suficientes para gerar camadas de aproximadamente $80 \AA$ Å de cada lado. A seguir, foi adicionado ao sistema o peptídeo de modo que a menor distância entre qualquer um de seus átomos e a posição média dos átomos de fósforo dos fosfolipídios mais próximos fosse $20 \AA$. Esses sistemas contendo a bicamada (pura ou mista), as camadas de água e o peptídeo posicionado na solução foram utilizados como arquivos de entrada para o script. Aplicamos o script para a geração de 60 janelas que diferiram na distância do peptídeo ao centro da bicamada. Selecionamos três dessas configurações, para cada composição da bicamada, para realizarmos as análises apresentadas neste trabalho, sendo que em cada uma delas o peptídeo ocupa ambientes físico-químicos distintos: no sistema 1 o peptídeo está na solução, com seu centro de massa a uma distância em z de aproximadamente $50 \AA \AA$ do centro da bicamada, no sistema 2 o peptídeo se localiza na interface água/bicamada estando seu centro de massa a aproximadamente $20 \AA$ do centro da bicamada e no sistema 3 o peptídeo está completamente inserido na bicamada com o centro de massa coincidindo com o centro da bicamada.

$\mathrm{Na}$ figura 1 apresentamos os sistemas gerados com a bicamada pura e na figura 2 os sistemas gerados com a bicamada mista. As moléculas de água estão representadas como uma superfície roxa, o peptídeo representado em licorice e com os resíduos coloridos conforme sua natureza física, em azul e vermelho resíduos com carga positiva ou negativa, respectivamente, em verde os 
polares e em branco os hidrofóbicos. Na bicamada, os grupos acílicos das moléculas de POPC estão coloridos em ciano e das de POPG em vermelho, nas região polar os átomos de fósforo são as esferas marrons, os nitrogênios esferas azuis e os oxigênios esferas vermelhas. Observamos que em todos os casos o peptídeo está inserido adequadamente na bicamada sem alterações conformacionais significativas tanto no peptídeo como na bicamada. Não observamos superposições severas que podem levar, quando da simulação desses sistemas, a valores de energia que possam desestabiliza-los.
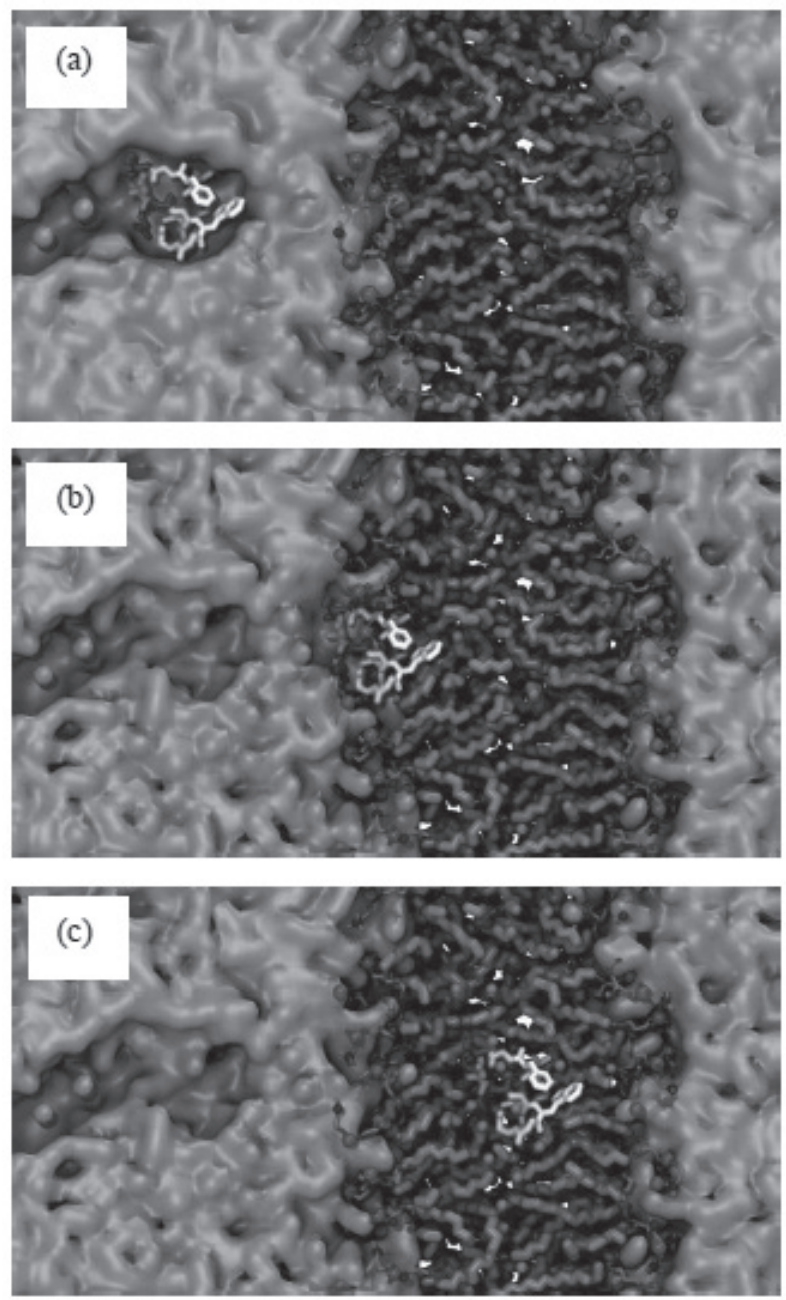

Figura 1. Sistemas gerados com a bicamada de POPC utilizando o método desenvolvido: (a) com o peptídeo localizado na solução. (b) com o peptídeo localizado na interface água/bicamada. (c) com o peptídeo localizado no interior da bicamada.
A figura 3 mostra o RMSD entre as posições dos átomos de fósforo da conformação original e cada etapa do processo de fechamento da bicamada descrito nos itens 5 e 6 da seção de métodos. Nas configurações analisadas nesse trabalho esse processo aconteceu apenas para os sistemas 2 e 3 . Observa-se na figura que os sistemas 2 (linha preta) e 3 (linha verde) da bicamada pura apresentam resultados semelhantes com o valor mínimo para o RMSD por volta de 0,5 A. O mesmo acontece para os sistemas contendo a bicamada mista, como podemos verificar na figura, os
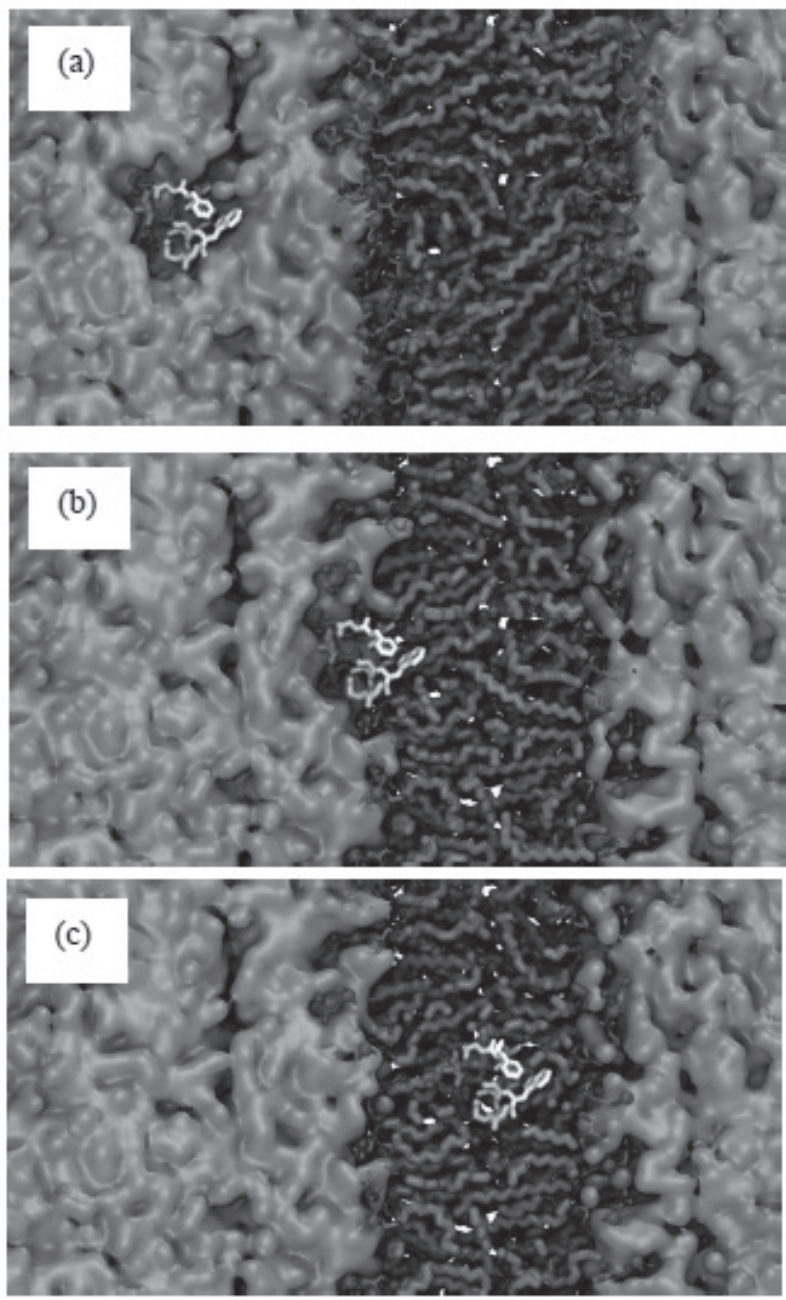

Figura 2. Sistemas gerados com a bicamada de POPC e POPG utilizando o método desenvolvido: (a) com o peptídeo localizado na solução. (b) com o peptídeo localizado na interface água/bicamada. (c) com o peptídeo localizado no interior da bicamada. 
sistemas 2 (linha vermelha) e 3 (linha azul) apresentam valor mínimo de RMSD em torno de $0,1 \AA$ alcançado com menos passos. Esses valores de RMSD menores que $1 \AA$ apontam que em ambos os casos (bicamada pura e simples) a configuração estrutural da bicamada obtida após o processo de inserção do peptídeo é muito próxima da observada na bicamada original que estava equilibrada. Assim, os sistemas gerados pelo nosso método irão requerer muito menos tempo de equilibração que sistemas gerados por métodos onde se exclui grande quantidade de fosfolipídeos para a inserção da proteína.

Por meio de uma varredura ao longo do eixo z (perpendicular a membrana) pode-se calcular a densidade de massa referente às moléculas de interesse em cada ponto do sistema, este mapeamento fornece o Perfil de Densidade de Massa. Com isso, é possível determinar a região média ocupada pelo solvente, pela bicamada e pela proteína, além de permitir estimar a espessura média da bicamada.

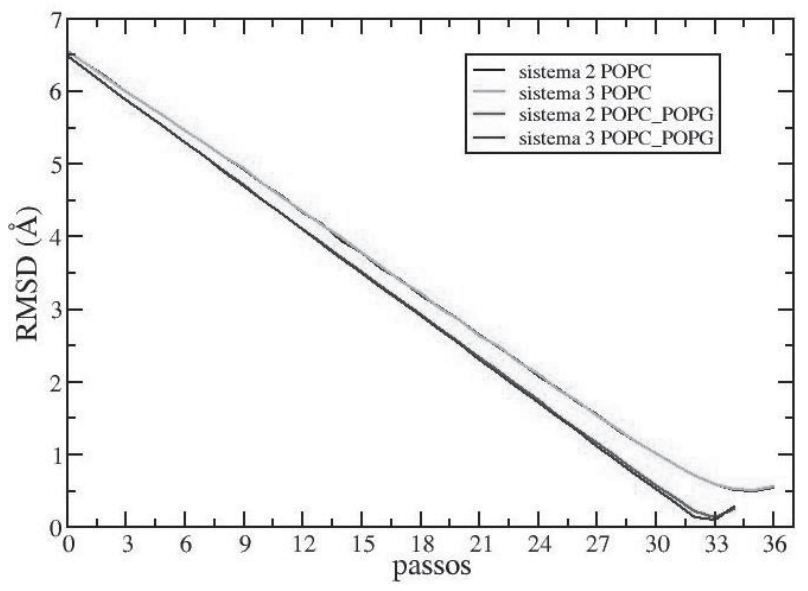

Figura 3. RMSD entre as posições dos átomos de fósforo da bicamada original e a bicamada no processo de fechamento após a inserção do peptídeo. São apresentados os resultados apenas para sistemas onde foi necessário abrir a bicamada para a inserção do peptídeo, no caso os sistemas 2 e 3 das bicamadas pura e mista. As linhas preta e verde representam os sistemas 2 e 3 da bicamada pura, respectivamente. As linhas vermelha e azul representam os sitemas 2 e 3 da bicamada mista.
Os gráficos do Perfil de Densidade de Massa (PDM) dos sistemas contendo bicamada de POPC (bicamada pura) e de POPC com POPG (bicamada mista) são mostrados nas figuras 4 e 5, respectivamente. Analisando as linhas vermelhas (PDM dos fosfolipídeos) e azuis (PDM do grupo fosfato) de todos os gráficos observamos que ambas as bicamadas apresentam uma espessura média em torno de $40 \AA$ mesmo após o processo de abertura e fechamento para a inserção do peptídeo. Isso mostra que o método aqui descrito perturba muito pouco as bicamadas com relação a sua espessura. Observando as linhas verdes vemos onde o peptídeo se localiza, em média, em cada sistema. O PDM da água indica coexistência com a região polar da bicamada na interface e é nulo na região no centro da membrana, como esperado.

\section{Conclusão}

A partir dos resultados e discussões realizadas acima, podemos concluir que o método proposto e o script implementado são eficientes para a inserção de proteínas em bicamadas lipídicas causando a mínima perturbação possível. O RMSD calculado mostra que a diferença entre as posições dos átomos de referência (fósforos) da bicamada original e da bicamada gerada ficou abaixo de $1 \AA$, com convergência mais rápida para a bicamada mista (POPC/POPG).

Através do gráfico do Perfil de Densidade de Massa observamos que a espessura da bicamada fica em torno de $40 \AA$ em todos os sistemas, o que mostra que o método de inserção da proteína pouco altera a estrutura da bicamada. O PDM também nos mostra que nosso método também causa baixo ou nenhum impacto na solvatação da bicamada, já que a distribuição das moléculas de água permanece inalterada depois do processo de fechamento da bicamada.

\section{Perpectivas Futuras}

Os próximos passos serão a execução de simulações de Dinâmica Molecular dos sistemas gerados para o monitoramento de propriedades físico-químicas da bicamada como área por lipídeo, PDM e parâmetro de ordem. Observaremos quanto tempo de simulação será necessário para alcançarmos valores de referência e 

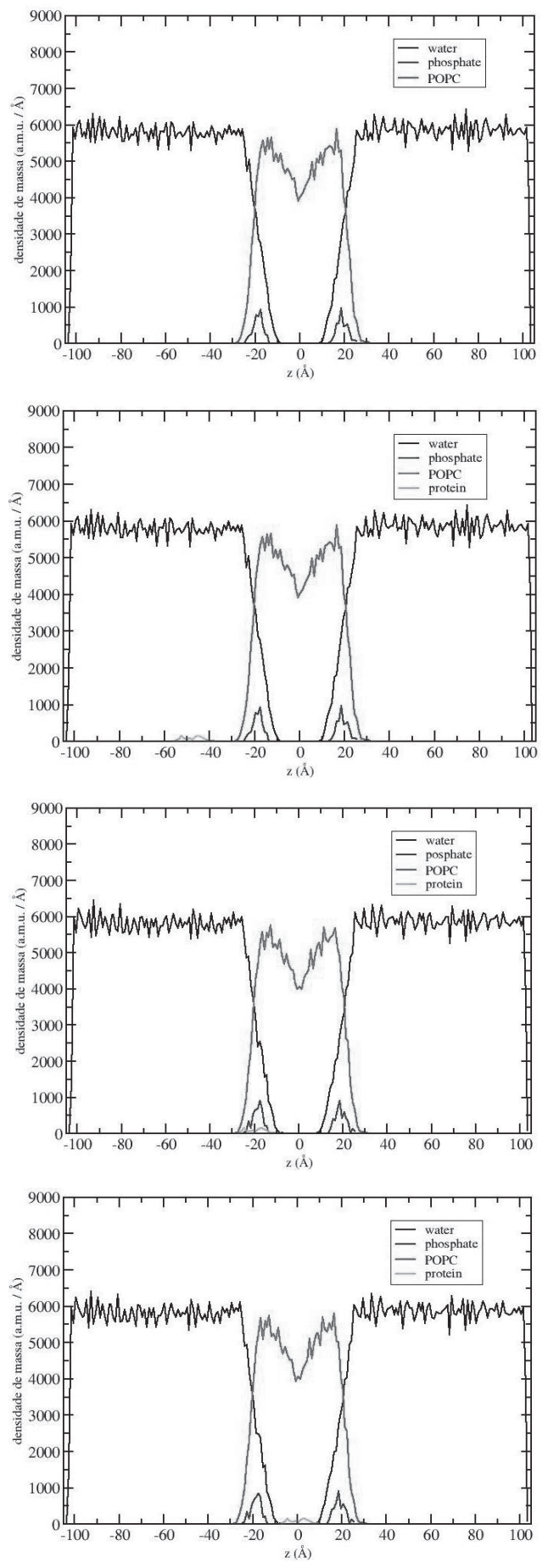

Figura 4. Perfil de Densidade de Massa (PDM) da bicamada pura de POPC em relação ao eixo z, onde em (a), (b), (c) e (d) a linha preta representa a água, a linha azul os grupos fosfato dos fosfolipídeos, a linha vermelha o POPC e a verde o peptídeo.
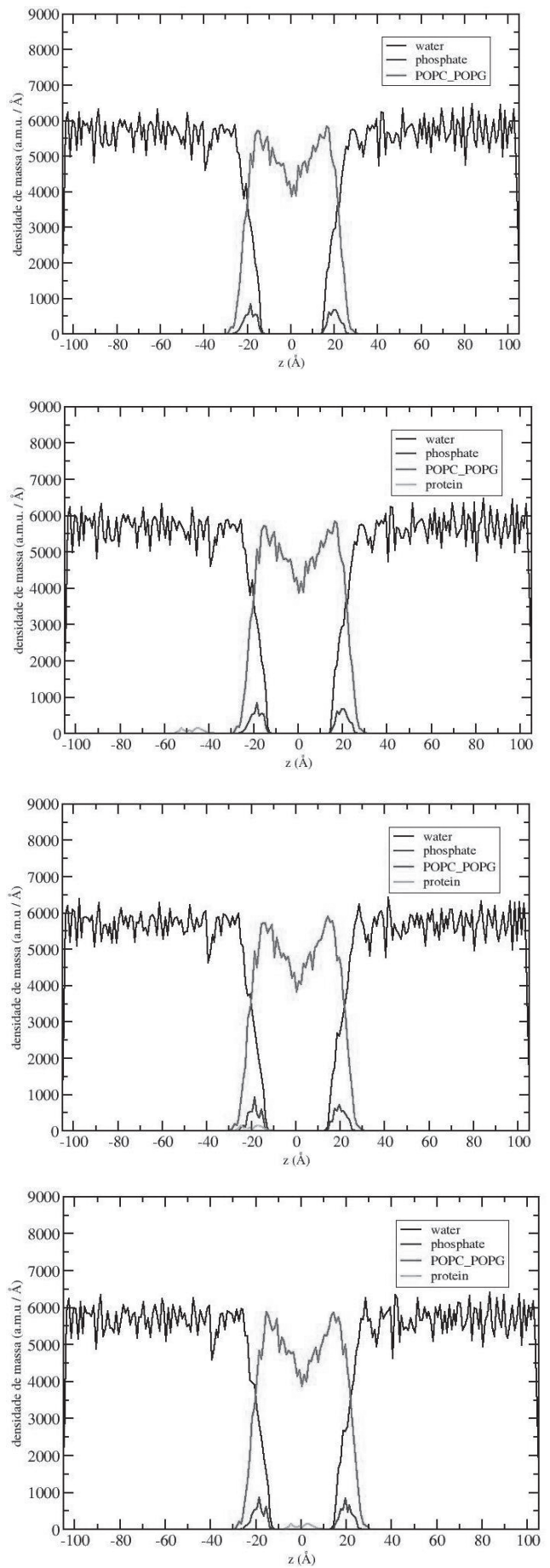

Figura 5. Perfil de Densidade de Massa (PDM) da bicamada mista de POPC e POPG em relação ao eixo z, onde em (a), (b), (c) e (d) a linha preta representa a água, a linha azul os grupos fosfato dos fosfolipídeos, a linha vermelha o POPC ou POPG e a verde o peptídeo. 
compararemos esses tempos com tempos obtidos a partir de simulações com sistemas gerados por outros métodos. Com isso teremos base para comparação de nosso método com outros já estabelecidos.

\section{Agradecimentos}

À Fapesp pelo apoio financeiro: processo $\mathrm{n}^{\circ}$ 2010/18169-3, Fundação de Amparo à Pesquisa do Estado de São Paulo (FAPESP).

Esta pesquisa tornou-se possível graças aos recursos computacionais disponibilizados pelo Núcleo de Computação Científica (NCC/GridUNESP) da Universidade Estadual Paulista (UNESP)

Pesquisa desenvolvida com o auxílio do CENAPADSP (Centro Nacional de Processamento de Alto Desempenho em São Paulo), projeto UNICAMP / FINEP - MCT.

\section{Referências}

1. Kandt, C., Ash, W. L. \& Peter Tieleman, D. Setting up and running molecular dynamicssimulations of membrane proteins. Methods 41, 475-488 (2007).

2. Humphrey, W., Dalke, A. \& Schulten, K. VMD: Visual molecular dynamics. J. Mol. Graph. 14, 33-\& (1996).

3. Phillips, J. C. et al. Scalable molecular dynamics with NAMD. J. Comput. Chem. 26, 1781-1802 (2005).

4. Giorgino, T. Computing 1-D atomic densities in macromolecular simulations: The density profile tool for VMD. Comput. Phys. Commun. 185, 317-322 (2014).

5. Wu, E. L. et al. CHARMM-GUI Membrane Builder toward realistic biological membrane simulations. J. Comput. Chem. 35, 1997-2004 (2014).

6. Brooks, B. R. et al. CHARMM: the biomolecular simulation program. J. Comput. Chem. 30, 1545-1614 (2009).

7. Jo, S., Kim, T. \& Im, W. Automated Builder and Database of Protein/Membrane Complexes for Molecular Dynamics Simulations. PLoS ONE 2, e880 (2007).

8. Jo, S., Lim, J. B., Klauda, J. B. \& Im, W. CHARMM-GUI Membrane Builder for Mixed Bilayers and Its Application to Yeast Membranes. Biophys. J. 97, 50-58 (2009).

\section{Luiz Fernando da Costa Zonetti ${ }^{a, b^{*}}$ \& Alexandre Suman de Araújo ${ }^{a}$}

\author{
a Universidade Estadual Paulista "Júlio de Mesquita Filho", São José \\ do Rio Preto /SP \\ ${ }^{\mathrm{b}}$ Instituto Federal de Educação, Ciência e Tecnologia de São Paulo - \\ Campus Birigui, Birigui/SP \\ *E-mail: luizfz@hotmail.com
}

